\title{
Endoscopic management of an esophagopleural fistula resulting from a gunshot wound
}
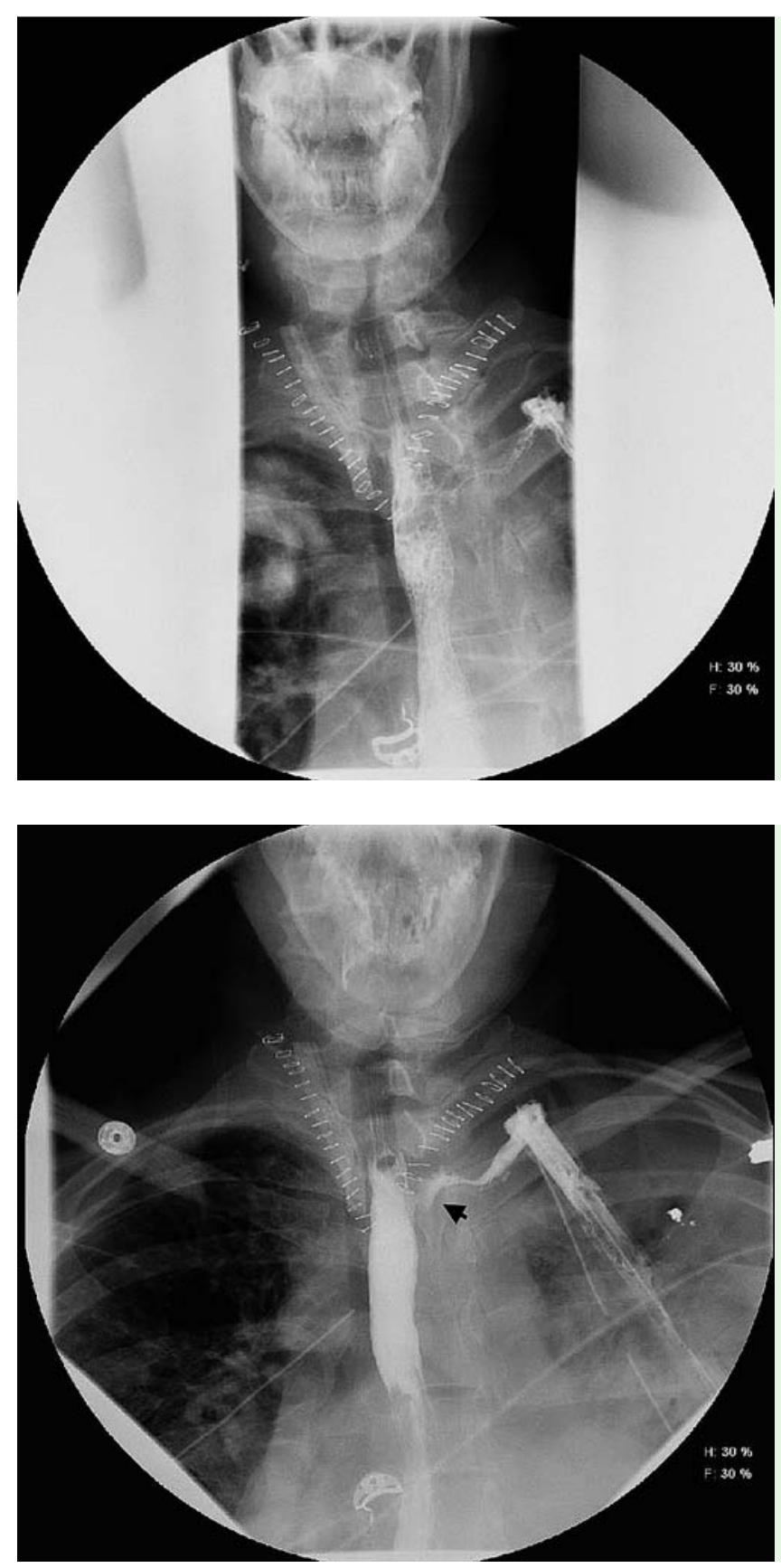

A 36-year-old man presented to the emergency department with a gunshot injury to his neck. He was intubated and taken to the operating room. As his neck was being dissected, an air leak in the trachea, indicative of a direct injury from the gunshot wound, was detected. This was repaired successfully. No damage to the esophagus was seen during an intraoperative esophagoscopy.
Fig. 1 Gastrografin esophagogram 6 days after surgery to the neck for a gunshot wound showing no evidence of an esophageal leak.

Fig. 2 Barium swallow performed the following day showing a well formed fistulous track from the esophagus to the left pleural space (arrow).

A Gastrografin esophagogram was done on the sixth postoperative day, before the patient recommenced oral intake, and did not show any esophageal leak ( $\bullet$ Fig. 1). However, within 1 day of his restarting oral intake, the patient developed a new onset leukocytosis. A barium swallow on the seventh postoperative day showed an esophageal fistula draining into the left pleural space ( Fig.2). This fistulous

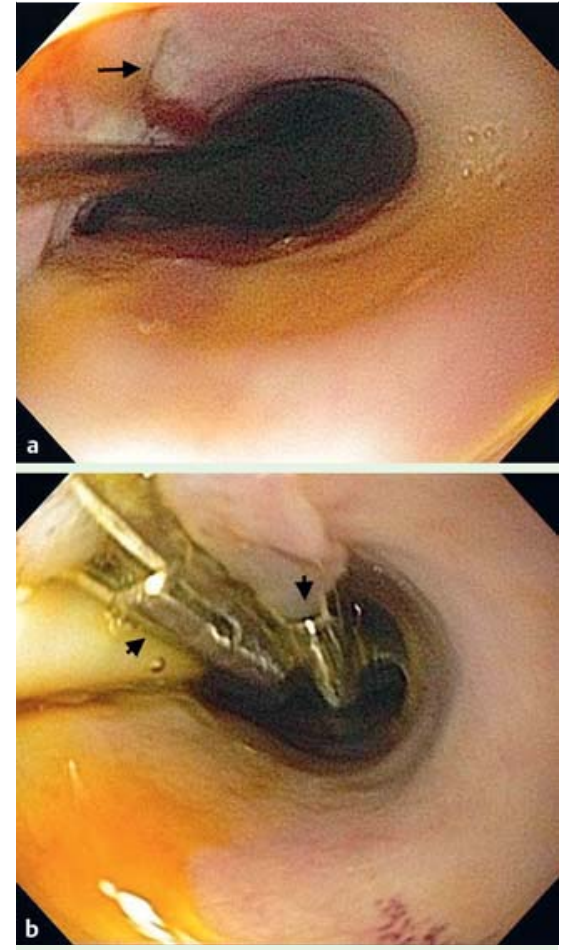

Fig.3 Views during esophagoscopy showing: a the opening of the esophageal fistula (arrow); b endoclips placed to close the esophageal fistula (arrows).

track was not seen on the preoperative esophagoscopy and it most likely developed postoperatively.

The fistulous track was well formed but it was considered to be very likely to close with nonoperative management. Therefore, an upper gastrointestinal endoscopy was performed which showed an $8-\mathrm{mm}$ esophageal fistula, $17 \mathrm{~cm}$ from the incisors, that was seen as soon as the upper esophageal sphincter was traversed with the endoscope ( $\nabla$ Fig. 3 a). The fistula was closed using three endoscopic Hemoclips (Wilson-Cook Medical Inc., WinstonSalem, North Carolina, USA) ( Fig.3b). A follow-up barium swallow 3 days later showed complete closure of the esophagopleural fistula ( Fig.4). The patient tolerated his oral diet well and he was discharged 12 days after the endoscopic closure.

This case illustrates some unique clinical features. First, the fistula formation was thought to be secondary to thermal injury to the esophagus from the bullet frag- 


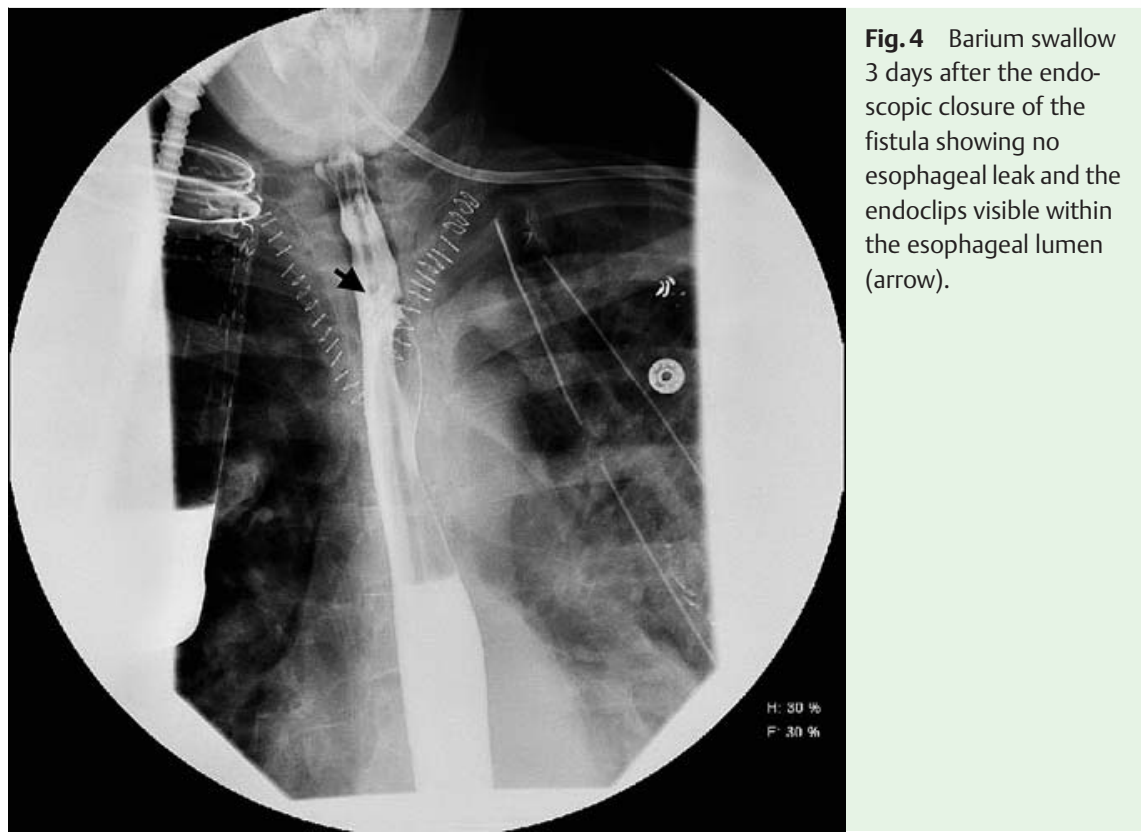

ments. The Gastrografin study failed to demonstrate the presence of the fistulous track, although Gastrografin studies are associated with a false-negative rate of up to $22 \%$ for the detection of traumatic esophageal fistulas [1]. Finally, the fistula size was small, which therefore permitted closure with endoclips. In conclusion, esophageal fistulas can form late after a

\section{Shashank Garg', Mark Katlic ${ }^{2}$,} Sudhir Dutta ${ }^{3}$

${ }^{1}$ Department of Internal Medicine, Sinai Hospital of Baltimore, Baltimore, Maryland, USA

${ }^{2}$ Department of Surgery, Sinai Hospital of Baltimore, Baltimore, Maryland, USA

${ }^{3}$ Division of Gastroenterology, Department of Medicine, Sinai Hospital of Baltimore and University of Maryland School of Medicine, Baltimore, Maryland, USA

\section{Reference}

1 Buecker A, Wein BB, Neuerburg JM et al. Esophageal perforation: comparison of use of aqueous and barium-containing contrast media. Radiology 1997; 202: 683-686

Bibliography

Dol http://dx.doi.org/

10.1055/s-0034-1365784

Endoscopy 2014; 46: E508-E509

(c) Georg Thieme Verlag KG

Stuttgart · New York

ISSN 0013-726X

and endoscopic closure of such fistulas is possible in carefully selected patients using commonly available endoclips.

Endoscopy_UCTN_Code_CCL_1AB_2AC_3AH

Competing interests: None

\section{Corresponding author \\ Sudhir Dutta, MD}

Division of Gastroenterology,

Department of Medicine, Sinai Hospital of Baltimore and University of Maryland School of Medicine, Baltimore

2411 W. Belvedere Ave, Suite 305

Baltimore

MD 21215

USA

Fax: +1-410-601-5757

sudhirdutta@hotmail.com 